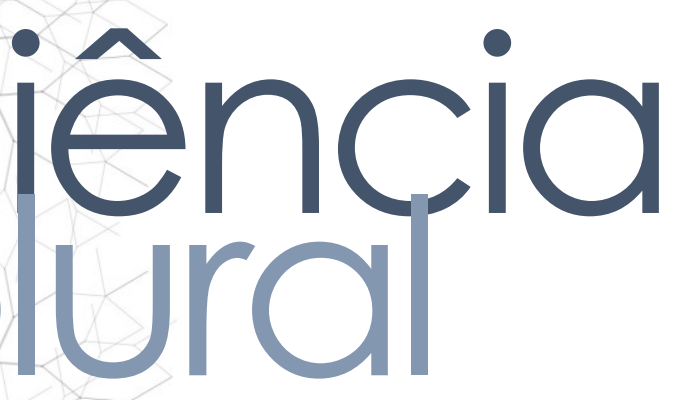

\title{
SERVIÇO DE SAÚDE ESCOLAR: EVIDÊNCIAS DA LITERATURA
}

\section{School health service: evidence from the literature}

\section{Servicio de salud escolar: evidencia de la literatura}

Karla Brandão de Araújo • Mestranda do Programa de Pós-graduação em Enfermagem em Saúde Pública da Universidade do Estado do Amazonas • Especialista em Obstetrícia e Vigilância Sanitária • Enfermeira do Instituto Federal de Educação, Ciência e Tecnologia do Amazonas • E-mail: enfufam@hotmail.com

Erika Oliveira Abinader $\bullet$ Mestre em Biologia Urbana Especialista Medicina do Trabalho • Médica do Instituto Federal de Educação, Ciência e Tecnologia do Amazonas• E-mail: erikabinader@ifam.edu.br

Vanessa Brandão de Araújo • Graduação em Educação Física • Especialista em Vigilância Sanitária • E-mail: nebrandao@gmail.com.br

Jamilly Brandão de Castro • Graduação em Enfermagem pela Unifor • E-mail:kaua3@yahoo.com.br

Karem de Souza Brandão • Graduação em Nutrição • Nutricionista do Instituto Federal de Educação, Ciência e Tecnologia do Amazonas • E-mail: karem.brandao@ifam.edu.br

Autora correspondente:

Karla Brandão de Araújo • E-mail: enfufam@hotmail.com 


\section{RESUMO}

Introdução: Ações em saúde, no âmbito escolar, pré-dispõe os indivíduos a adotarem medidas de proteção quanto a sua saúde reduzindo fatores de risco na vida adulta corroborando para melhor qualidade de vida. Objetivo: Pesquisar as evidências científicas sobre as ações de saúde ofertadas no ambiente escolar. Metodologia: Estudo descritivo, com abordagem qualitativa que através de revisão integrativa da literatura investigou as evidências sobre o objeto deste estudo utilizando as bases: LILACS e SCIELO. A coleta dos dados ocorreu em julho de 2020, aos pares, considerando o recorte temporal de cinco anos (2015 à 2020) nos idiomas português e inglês. A estratégia de busca foi formulada utilizando os descritores controlados e não controlados "serviços de saúde escolar", "promoção da saúde", "saúde na escola" e "saúde dos estudantes" que foram combinados entre si por meio dos operadores booleanos AND e OR. Resultados: Foram encontrados 1603 artigos que após uso de critérios de elegibilidade, restaram 05 na composição da amostra. Da análise do conteúdo foi evidenciado as lacunas quanto promoção da saúde no ambiente escolar. Conclusões: os serviços de saúde no âmbito escolar são realizados através de ações pontuais, geralmente motivadas por ações sanitaristas com modelo campanhista. Foi evidenciado a necessidade de educação permanente para capacitação dos profissionais.

Palavras-Chave: Serviços de saúde escolar, Promoção da Saúde, Equipe de assistência ao paciente.

\section{ABSTRACT}

Introduction: Health actions, in the school context, predispose individuals to adopt protective measures regarding their health, reducing risk factors in adulthood, corroborating for a better quality of life. Objective: To research the scientific evidence on health actions offered in the school environment. Methodology: Descriptive study with a qualitative approach that, through an integrative literature review, investigated the evidence on this study's object using the bases: LILACS and SCIELO. Data collection took place in July 2020, in pairs, considering the five-year time frame (2015 to 2020) in Portuguese and English. The search strategy was formulated using the controlled and uncontrolled descriptors "school health services", "health promotion", "health at school" and "student health" which were combined through the Boolean operators AND and OR. Results: 1603 articles found that five remained in the sample composition after using the eligibility criteria. The content analysis revealed the gaps regarding health promotion in the school environment. Conclusions: health services at school are carried out through specific actions, usually motivated by sanitary actions with a campaign model. The need for continuing education to train professionals was videnced.

ywords: School health services, Health Promotion, Patient care team. 


\section{RESUMEN}

Introducción: Las acciones de salud, en el contexto escolar, predisponen a los individuos a adoptar medidas protectoras respecto a su salud, reduciendo los factores de riesgo en la edad adulta, corroborando para una mejor calidad de vida. Objetivo: Investigar la evidencia científica sobre las acciones de salud que se ofrecen en el ámbito escolar. Metodología: Estudio descriptivo con enfoque cualitativo que mediante una revisión integradora de la literatura investigó la evidencia sobre el objeto de este estudio utilizando las bases: LILACS y SCIELO. La recolección de datos se realizó en julio de 2020, por parejas, considerando el marco temporal de cinco años (2015 a 2020) en portugués e inglés. La estrategia de búsqueda se formuló utilizando los descriptores controlados y no controlados "servicios de salud escolar", "promoción de la salud", "salud escolar" y "salud del estudiante" que se combinaron entre sí a través de los operadores booleanos AND y OR. Resultados: se encontraron 1603 artículos que, luego de aplicar los criterios de elegibilidad, quedaron 5 en la composición de la muestra. El análisis de contenido reveló las brechas en la promoción de la salud en el entorno escolar. Conclusiones: los servicios de salud en la escuela se realizan a través de acciones específicas, generalmente motivadas por acciones sanitarias con modelo de campaña. Se evidenció la necesidad de educación continua para formar profesionales.

Palabras clave: Servicios de salud escolar, Promoción de la salud, Equipo de atención al paciente. 


\section{ciêncíncia
puúl}

\section{Introdução}

Empoderar o indivíduo para adoção de hábitos de vida saudável possibilita o fortalecimento de potencialidades para o autocuidado. É importante fomentar esses hábitos desde a idade escolar para que tal atitude se perpetue na fase adulta. Em 2007 foi implementada a Política Pública denominada Programa Saúde na Escola (PSE) que visa a integração entre educação e saúde, na rede pública de ensino, para superação de vulnerabilidades que comprometam o pleno desenvolvimento de jovens e crianças $^{1}$.

As ações fomentadas no PSE são voltadas à prevenção, atenção e promoção em saúde. Estas vinculadas à Estratégia Saúde da Família. Para a implementação dessa integração, é considerado o contexto social e operacional das unidades de ensino respeitando seu projeto político pedagógico. Cinco são os componentes do PSE: avaliação da condição de saúde, atividades de promoção e prevenção, educação permanente dos profissionais, avaliação e monitoramento contínuo da condição de saúde e do programa ${ }^{1}$.

No contexto escolar, também, são ofertadas ações de saúde independente do PSE. A exemplo, algumas instituições Federais de ensino ofertam assistência ambulatorial aos discentes com abordagem semelhante nas dimensões da promoção, prevenção e assistência direta ${ }^{2}$. Desta intersetorialidade, emerge a disseminação de saberes advindas da interação do senso crítico com o comum para o pleno compartilhamento de informações que reflitam em comportamentos positivos de saúde e desenvolvimento humano ${ }^{3}$.

Na perspectiva da corresponsabilização intersetorial saúde-escola, é necessário definir o papel de cada entidade para que se possa construir um processo onde as peculiaridades e prioridades de cada instituição seja implementada sem interferir egativamente sobre a outra ${ }^{4}$.

Enquanto ferramenta transformadora da realidade, a educação em saúde lementada do contexto escolar constrói e reconstrói o saber sistematizado, 
harmônico e integral potencializando atitudes individuais e coletivas que corroboram para mudanças que minimizam situações de risco e fortalecem adoção de medidas de proteção contribuindo para melhoria da qualidade de vida.

Destarte, questiona-se: quais ações de saúde têm sido desenvolvidas no contexto escolar? Em busca da resposta desta pergunta norteadora, este trabalho visa pesquisar as evidências científicas sobre as ações de saúde ofertadas no ambiente escolar independente de vínculo à política pública.

\section{Metodologia}

Estudo de revisão integrativa da literatura que busca sintetizar estudos que abordem as ações de saúde desenvolvidas no ambiente escolar. Trata-se do levantamento de trabalhos que abordam a temática de interesse preenchendo lacunas e agrupando o que se tem publicado considerando as evidências científicas ${ }^{5}$.

O interesse pela temática originou-se do modelo teórico conceitual (Figura 1) elaborado pelos autores onde foi levantado o questionamento acerca das atividades assistenciais desenvolvidas no âmbito escolar, estas implementadas por equipe multiprofissional, quanto a demanda para o desenvolvimento de ações de saúde.

Figura 1- Modelo Teórico Conceitual referente às demandas de ações em saúde escolar. Manaus-AM, 2020.

\section{EMERGÊNCIAS}

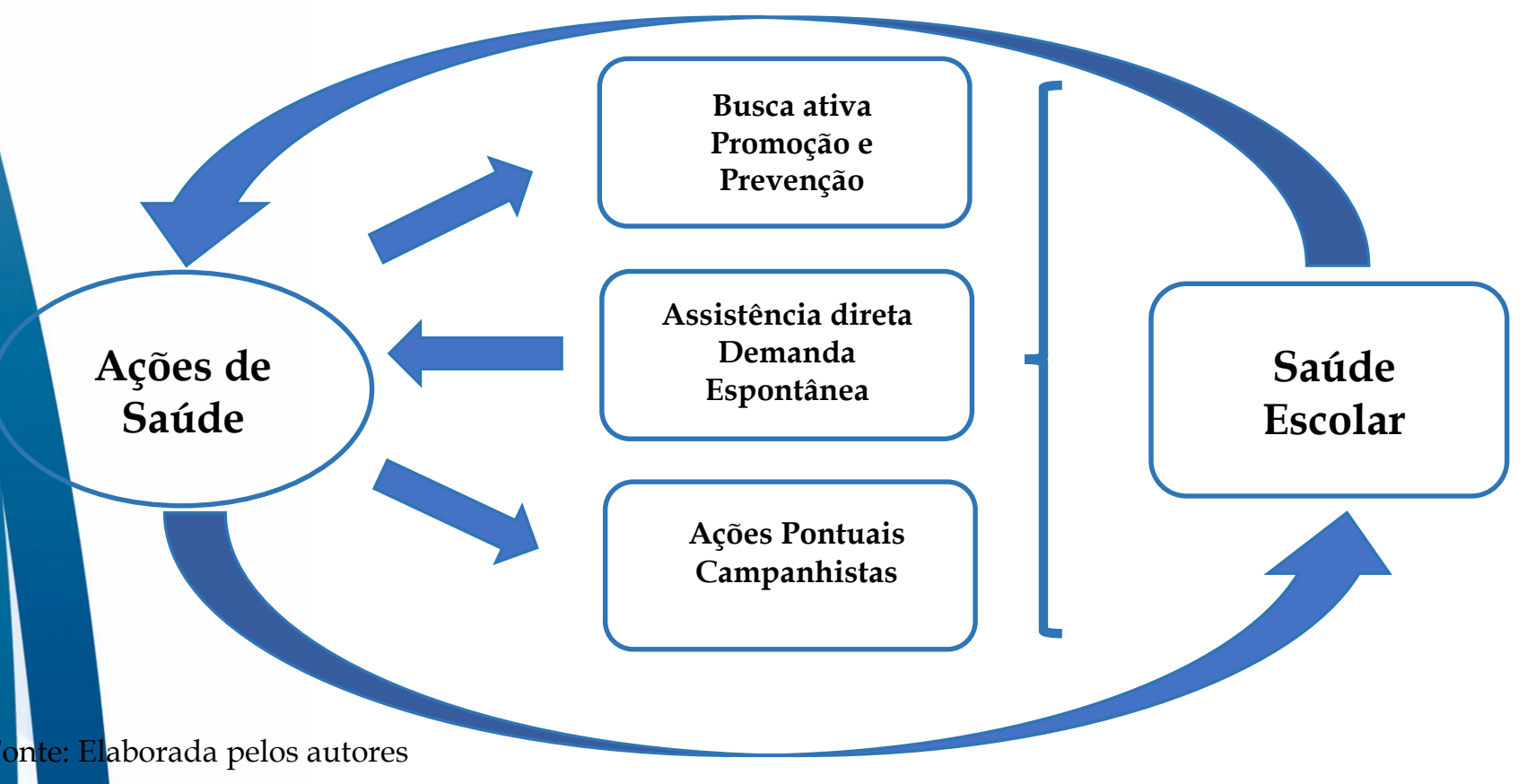


Através deste modelo gerou-se a hipótese de que as ações em saúde no âmbito escolar demandam da busca ativa, espontaneamente, por ações campanhistas e diretamente quando das situações de emergências.

A coleta dos dados foi efetivada aos pares, em julho de 2020, nas bases Literatura Latino-Americana e do Caribe em Ciências da Saúde (LILACS) e na Scientific Eletronic Library Online (SCIELO). Dos artigos selecionados, houve discordância em dois estudos quando da etapa de leitura do título e resumo. Porém, do consenso entre os pares e análise de um terceiro revisor, optou-se pela exclusão de ambos.

Originalmente foi incluída a Base de Dados de Enfermagem (BDENF), mas não foram encontrados estudos que atendessem aos critérios de elegibilidade para este estudo. Tais critérios foram: disponibilidade na íntegra online, recorte temporal correspondente ao período 2015 - 2020, nos idiomas inglês e português, região como assunto o Brasil e que tinham como temática principal os serviços de saúde escolar. Foram excluídas revisões, guias clínicos, screening studies, Evaluation studies. Quanto ao tipo de estudo foram eleitos os de prevalência, ensaio clínico, pesquisa de intervenção, estudos de incidência e pesquisas qualitativas.

Para formulação da estratégia de busca foram utilizados os descritores em saúde controlados e não controlados "serviços de saúde escolar", "promoção da saúde" ,"saúde na escola" e "saúde dos estudantes" combinados por meio dos operadores booleanos $A N D$ e OR. Com a estratégia foram identificados 1.603 estudos. Após aplicação dos critérios de elegibilidade, a amostra reduziu para 64 estudos. Com a leitura do título e resumo foram excluídos 59 trabalhos. Os remanescentes foram lidos na íntegra não gerando mais exclusões e constituindo a amostra final com 05 artigos, sendo quatro da LILACS e um da SCIELO. O fluxograma da seleção consta na figura 2. 
Figura 2- Fluxograma da seleção dos estudos. Manaus-AM, 2020.

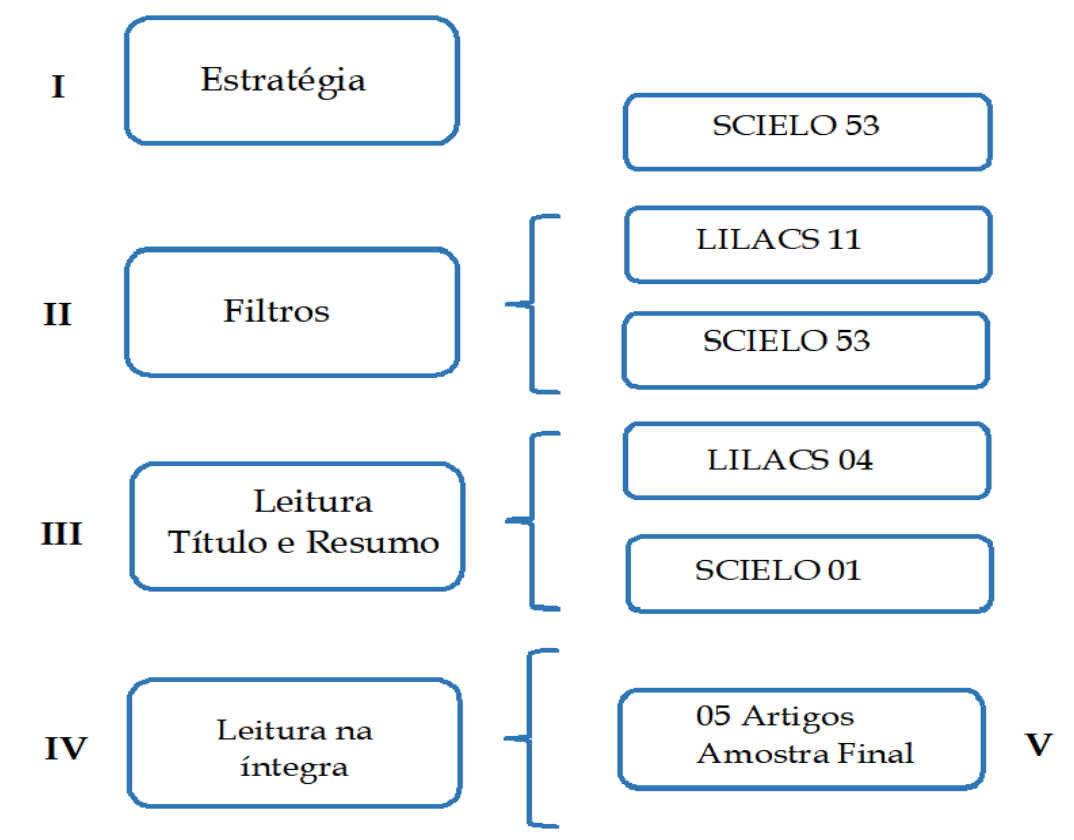

Fonte: Elaborada pelos autores

Os cinco estudos selecionados foram catalogados em tabela do programa Office 365 Excel ${ }^{\circledR}$ e sumarizados constando as categorias: título, autor, ano, país, base de dados, tipo de estudo e resultados (Tabela 1). Quanto ao nível de evidências foi adotado o proposto por Melnyk ${ }^{6}$ que varia de I à VII e quanto mais próximo de I, maior a força da evidência. Sendo: I- revisões sistemáticas, metanálise; II- ensaio clínico randomizado; III- ensaio clínico sem randomização; IV- coorte, caso-controle; Vrevisão sistemática de trabalhos descritivos; VI- estudo único descritivo ou qualitativo; VII- opinião de comitês de especialistas ou autoridades. Não foi necessário anuência por comitê de ética por tratar-se de uma revisão da literatura que utilizou fonte de dados de domínio público.

\section{Resultados e Discussão}

A seleção final foi composta por artigos com baixo nível de evidência, nível VI, demonstrando a necessidade de publicações com melhores evidências. Na base de ados LILACS está indexado 80\% dos artigos selecionados. Dos periódicos, os estudos ontram-se na Revista Brasileira de Materno Infantil9 , Revista Ciência Plural7,8, ista de Saúde Coletiva ${ }^{10}$ e na Revista Brasileira de Enfermagem ${ }^{11}$. 
Quanto ao delineamento dos estudos, foram identificados três descritivos, um seccional com abordagem quantitativa e um qualitativo. Com relação ao recorte temporal, os estudos são bem atuais prevalecendo publicações do ano de 2020. Os estudos foram sumarizados e constam no quadro 1.

Quadro 1- Sumarização dos artigos selecionados. Manaus-AM, 2020.

\begin{tabular}{|c|c|c|c|c|c|}
\hline & $\begin{array}{c}\text { Autores } \\
\text { Ano } \\
\text { País } \\
\end{array}$ & Título & $\begin{array}{l}\text { Base } \\
\text { de } \\
\text { dados }\end{array}$ & $\begin{array}{l}\text { Tipo de } \\
\text { Estudo }\end{array}$ & Resultados \\
\hline $\mathbf{A 1}^{7}$ & $\begin{array}{c}\text { Suassuna et al. } \\
2020 \\
\text { Brasil }\end{array}$ & $\begin{array}{l}\text { Percepções de alunos da } \\
\text { rede pública de ensino de } \\
\text { Natal/RN sobre educação } \\
\text { em saúde na escola. }\end{array}$ & LILACS & $\begin{array}{c}\text { Seccional } \\
\text { Quantitativo }\end{array}$ & $\begin{array}{l}\text { Na percepção dos alunos } \\
\text { não é percebido ações de } \\
\text { promoção da saúde nas } \\
\text { escolas, sendo necessário } \\
\text { redirecionar as ações. }\end{array}$ \\
\hline $\mathrm{A} 2^{8}$ & $\begin{array}{l}\text { Oliveira e } \\
\text { Machado } \\
2020 \\
\text { Brasil }\end{array}$ & $\begin{array}{l}\text { Percepção dos profissionais } \\
\text { da Estratégia Saúde da } \\
\text { Família sobre os processos } \\
\text { educativos em saúde }\end{array}$ & LILACS & Qualitativo & $\begin{array}{l}\text { Os profissionais } \\
\text { reconhecem a necessidade } \\
\text { de adequações nas } \\
\text { estratégias desenvolvidas } \\
\text { e que necessitam de } \\
\text { capacitação para tal. }\end{array}$ \\
\hline$A 3^{9}$ & $\begin{array}{c}\text { Bezerra et al. } \\
2017 \\
\text { Brasil }\end{array}$ & $\begin{array}{l}\text { Saúde e nutrição em escolas } \\
\text { públicas e privadas de } \\
\text { Recife. }\end{array}$ & LILACS & Descritivo & $\begin{array}{l}\text { Ações de saúde e nutrição } \\
\text { são implementadas } \\
\text { satisfatoriamente em } \\
\text { escolas públicas. O mesmo } \\
\text { não ocorre na rede } \\
\text { privada. }\end{array}$ \\
\hline A4 ${ }^{10}$ & $\begin{array}{l}\text { Carvalho } \\
2015 \\
\text { Brasil }\end{array}$ & $\begin{array}{l}\text { A saúde vai à escola: a } \\
\text { promoção da saúde em } \\
\text { práticas pedagógicas. }\end{array}$ & LILACS & Descritivo & $\begin{array}{l}\text { Ações de saúde alteram a } \\
\text { dinâmica escolar. }\end{array}$ \\
\hline A511 & $\begin{array}{l}\text { Ligia et al. } \\
2019 \\
\text { Brasil }\end{array}$ & $\begin{array}{lrr}\text { A saúde } & \text { na } & \text { escola: } \\
\text { percepções } & \text { do } & \text { ser } \\
\text { adolescente. } & & \end{array}$ & SCIELO & Descritivo & $\begin{array}{l}\text { A saúde escolar envolve } \\
\text { ações higienistas e } \\
\text { assintencialialista. }\end{array}$ \\
\hline
\end{tabular}

Fonte: Elaborada pelos autores

O estudo A1 descreve as percepções de alunos da rede pública do ensino médio, em um estado do nordeste, quanto as ações de educação em saúde ofertadas no mbiente escolar. Conclui que tais ações são incipientes e que os alunos reconhecem a importância de sua implementação, bem como a necessidade de ações contínuas que erem vínculo com os profissionais da saúde para reduzir vulnerabilidades. É xpresso que a educação em saúde, quando ocorre, é realizada pelo docente da stituição denotando fragilidade na execução do que é preconizado pelo PSE. 
Em analogia ao estudo descrito anteriormente, Oliveira e Machado $^{8}$ (A2) retratam a percepção dos profissionais da Estratégia Saúde da Família (ESF) quanto ao processo educativo em saúde para adolescentes. Assim como em A1, os autores fazem referência ao PSE reconhecendo que ainda há muito a se avançar em estratégias pedagógicas e em educação permanente para se implementar o programa em sua integralidade. Das ações ofertadas, revelam dificuldade em desenvolver certas atividades em decorrência de barreiras culturais impostas pelos responsáveis quanto a algumas temáticas fato que interfere na efetividade e planejamento das ações.

A implementação de ações voltadas a promoção de hábitos alimentares saudáveis e a prática de atividade física foram objeto do estudo A3 que analisou tais ações na rede pública e privada, portanto pertencente e externo ao PSE. Esse paralelo auxilia na percepção se as ações são ofertadas por obrigatoriedade de uma política pública ou se parte da corresponsabilização intersetorial saúde-escola. Chegou-se à conclusão de que a escola é um ambiente que facilita a adoção de comportamentos saudáveis, porém existem barreiras institucionais e sociais que dificultam o engajamento da comunidade. Na rede pública, houve maior cobertura das ações em comparação à rede privada, porém, ainda aquém do desejável.

Para uma efetiva ação intersetorial da dicotomia saúde-escola é necessário a utilização de práticas pedagógicas por parte dos profissionais de saúde para que se reduza o caráter impositivo das ações de educação em saúde, esse é o posicionamento do estudo A4. Destaca-se a necessidade de se conhecer a realidade objetiva de cada sujeito para o correto direcionamento das ações. Ressaltando que a dinâmica escolar é alterada quando da implementação de ações em saúde e que, por vezes, o profissional da saúde não está preparado para interagir com o escolar.

Das possibilidades de ações ofertadas predominam as de caráter higienistas e assistenciais como as atividades de prática física e educação popular, esse foi o desfecho do estudo A4. Afirma que é necessário reorganizar a forma de se fazer ducação em saúde através da escuta qualificada para construção de um modelo que pare as necessidades e fragilidades do escolar. 


\section{ciência
piural}

Os estudos selecionados relatam a importância e as lacunas no fazer saúde escolar, porém não elencam as atividades desenvolvidas o que caracteriza a limitação do presente estudo. São unânimes em afirmar a necessidade de se conhecer o públicoalvo para subsidiar o desenvolvimento das atividades educativas e que o profissional da saúde, enquanto educador, deve procurar aperfeiçoar a prática didática para que se tenha êxito nas ações ofertadas.

Apenas um estudo relatou a saúde escolar independente do PSE o que denota que as ações, quando implementadas, são direcionadas de forma pontual para concretizar o que demanda a política. Embora não implicitamente expresso, os estudos revelam o caráter campanhistas e biomédico prevalentes.

A saúde escolar necessita de um processo participativo e dinâmico da interface educação-saúde. As temáticas violência, uso de substâncias psicoativas, obesidade, autocuidado são relevantes para sensibilizar a comunidade escolar na adoção de atitudes saudáveis. É necessário o planejamento coletivo para que o escolar sinta-se parte integrante e se corresponsabilize por sua saúde ${ }^{12}$.

$\mathrm{O}$ ambiente escolar se configura em potencial instrumentalizador para implementação de ações em saúde. Os profissionais de saúde, vinculados ou não ao PSE, partindo dos determinantes em saúde podem traçar estratégias que facilitem e orientem suas ações. O modelo teórico conceitual, outrora reportando pelos autores (Figura1), foi validado com os achados dos artigos selecionados.

\section{Conclusões}

Os serviços de saúde no âmbito escolar são realizados através de ações pontuais, geralmente motivadas por ações sanitaristas com modelo campanhista. É notório a necessidade de capacitação dos profissionais de saúde para atuarem na saúde escolar. Barreiras socioculturais dificultam a implementação das ações devido a

mitos e tabus enraizados nos responsáveis dos escolares o que limita a atuação integral cada no conceito ampliado de saúde. O PSE é uma Política Pública de grande loração e potencial para promoção da saúde, porém suas diretrizes devem incluir a sibilização da comunidade para compreensão de sua relevância. A rede privada de 
ensino, embora não inclusa no PSE, poderia implementá-la enquanto ferramenta para prática de ações educativas com vistas à promoção da saúde e qualidade de vida.

\section{Referências}

1.Ministério da Educação [Internet]. Programa saúde nas escolas [atualizado 2020 Jul 20; citado 2000 Ago 20]. Disponível em: http:/ / portal.mec.gov.br/expansao-da-redefederal/194-secretarias-112877938/ secad-educacao-continuada-223369541/14578programa-saude-nas-escolas. 2020.

2. Araujo KB de, Abinader EO, Brandao K de S, Xisto VH da S, Fleury S da S. Promovendo saúde em um Instituto Federal de Educação: relato de experiência. RSD. 2020 Jul [citado 2020 Ago 22]; 9(8): e631986071. Disponível em: https:/ / rsdjournal.org/index.php/rsd/article/view/6071

3. Paes CCDC, Paixão AN dos P. A importância da abordagem da educação em saúde: revisão de literatura. REVASF. 2016 Dez [citado 2020 Ago 23]; 6(11). Disponível em: https://www.periodicos.univasf.edu.br/index.php/revasf/article/view/38

4. Casemiro JP, Fonseca ABC, Secco FVM. Promover saúde na escola: reflexões a partir de uma revisão sobre saúde escolar na América Latina. Ciênc. saúde coletiva. 2014 Mar [citado 2020 Ago 22]; 19(3): 829-840. Disponível em: http:/ / www.scielo.br/scielo.php?script=sci_arttext\&pid=S1413$81232014000300829 \& \operatorname{lng}=$ en.

5. Creswell JW. Projeto de Pesquisa, métodos qualitativo, quantitativo e misto. Porto Alegre: Artmed; 2010.p-51.

6. Melnyk BM, Fineout-Overholt E. Making the case for evidence-based practice. In: Melnyk BM, Fineout-Overholt E. Evidence based practice in nursing \& healthcare. A guide to best practice. Philadelphia: Lippincot Williams \& Wilkins. 2005; 3-24.

7. Suassuna A, de Oliveira SF, Diniz PT, Christiane AMF. Percepções de alunos da rede pública de ensino de natal/RN sobre educação em saúde na escola. Rev. Ciênc. Plural. 2020 Jun [citado 2020 Ago 20]; 6(2):66-1. Disponível em: https:/ / periodicos.ufrn.br/rcp/article/view/19841

8. Oliveira SF, Machado FCA. Percepção dos profissionais da estratégia saúde da família sobre processos educativos em saúde. Revista Ciência Plural. 2020 Jun [citado 020 Ago 20]; 6(1):56-70. Disponível em:

ps:/ / periodicos.ufrn.br/rcp/article/view/18905/12529. 
9. Bezerra MA, Carvalho EF, Oliveira JS, Leal VS. Saúde e nutrição em escolas públicas e privadas da cidade do Recife. Rev. Bras. Saude Mater. Infantil. 2017 Mar [citado 2020 Ago 20]; 17(1): 191-200. Disponível em: http:/ / www.scielo.br/scielo.php?script=sci_arttext\&pid=S151938292017000100191\&lng=en.

10. Carvalho FFB. A saúde vai à escola: a promoção da saúde em práticas pedagógicas. Physis . 2015 Dec [citado 2020 Ago 20] ; 25( 4 ): 1207-1227. Disponível em: http:/ / www.scielo.br/scielo.php?script=sci_arttext\&pid=S010373312015000401207\&lng=en.

11. Ligia CM, Silva RMCRA, Pereira ER, Faial CSG. Saúde na escola: percepções de ser adolescente. Rev. Bras. Enferm. 2019 [citado 2020 Ago 20]; 73 (3): e20190068. Disponível em: http:/ / www.scielo.br/scielo.php?script=sci_arttext\&pid=S003471672020000300182\&lng=en. Epub em 22 de abril de 2020.

12. Giugliani C, Cesa KT, Flores EMTL, Mello Vânia Roseli de, Robinson Patrícia Genro. A escola como espaço de participação social e promoção da cidadania: a experiência de construção da participação em um ambiente escolar. Saúde debate. 2020 [citado 2020 Set 01] ; 44( spe1 ): 64-78. Disponível em:

http:/ / www.scielo.br/scielo.php?script=sci_arttext\&pid=S0103$11042020000500064 \& \operatorname{lng}=$ en. 